\title{
First microsatellite markers for the pine catkin sawfly Xyela concava (Hymenoptera, Xyelidae) and their application in phylogeography and population genetics
}

\author{
Dustin Kulanek $^{\text {Corresp., } 1}{ }^{1}$, Stephan M Blank ${ }^{1}$, Katja Kramp \\ ${ }^{1}$ Senckenberg Deutsches Entomologisches Institut, Müncheberg, Germany \\ Corresponding Author: Dustin Kulanek \\ Email address: dustin.kulanek@senckenberg.de
}

Microsatellites are widely used as powerful markers in population genetics because of their ability to access recent genetic variation and to resolve subtle population genetic structures. However, their development, especially for non-model organisms with no available genome-wide sequence data, has been difficult and time-consuming. Here, a commercial high-throughput sequencing approach (HTS) was used for the very first identification of microsatellite motifs in the genome of Xyela concava and the design of primer pairs flanking those motifs. Sixteen of those primer pairs were selected and implemented successfully to answer questions on the phylogeography and population genetics of $X$. concava. The markers were characterized in three geographically distinct populations of $X$. concava and tested for cross-species amplification in two additional Xyela and one Pleroneura species (Xyelidae). All markers showed substantial polymorphism as well as revealing subtle genetic structures among the three genotyped populations. We also analyzed a fragment of the nuclear gene region of sodium/potassium-transporting ATPase subunit alpha ( $\mathrm{NaK}$ ) and a mitochondrial gene region partly coding for cytochrome oxidase subunit I (COI) to demonstrate different genetic resolutions and sex-biased patterns of these markers, and their potential for combined use in future studies on the phylogeography and population genetics of $X$. concava. Although a limited number of populations was analyzed, we already obtained new insights on the latter two topics. The microsatellites revealed a generally high gene flow between the populations, but also suggested a deep historical segregation into two genetic lineages. This deep genetic segregation was confirmed by NaK. While the high gene flow was unexpected, because of assumed restricted dispersal ability of $X$. concava and the discontinuous distribution of the host trees between the populations, the segregation of two lineages is comprehensible and could be explained by different refuge areas of the hosts during glacial times. The $\mathrm{COI}$ results showed a discordant strong genetic structure between all populations, which might be explained by the smaller effective population size of the mitochondrial genome. 
However, given the frequent evidence of a similar nature in recent studies on sawflies, we also consider and discuss mitochondrial introgression on population level as an alternative explanation. 
1 First microsatellite markers for the pine catkin sawfly

2 Xyela concava (Hymenoptera, Xyelidae) and their

3 application in phylogeography and population

4 genetics

5

6 Dustin Kulanek ${ }^{1}$, Stephan M. Blank ${ }^{1}$, Katja Kramp ${ }^{1}$

7

$8{ }^{1}$ Senckenberg Deutsches Entomologisches Institut, Müncheberg, Germany

Corresponding Author:

Dustin Kulanek ${ }^{1}$

Eberswalder Str. 90, 15374 Müncheberg, Germany

14

Email address: Dustin.Kulanek@senckenberg.de 
15

16

17

18

19

20

21

22

23

24

25

26

27

28

\section{Abstract}

Microsatellites are widely used as powerful markers in population genetics because of their ability to access recent genetic variation and to resolve subtle population genetic structures. However, their development, especially for non-model organisms with no available genomewide sequence data, has been difficult and time-consuming. Here, a commercial high-throughput sequencing approach (HTS) was used for the very first identification of microsatellite motifs in the genome of Xyela concava and the design of primer pairs flanking those motifs. Sixteen of those primer pairs were selected and implemented successfully to answer questions on the phylogeography and population genetics of $X$. concava. The markers were characterized in three geographically distinct populations of $X$. concava and tested for cross-species amplification in two additional Xyela and one Pleroneura species (Xyelidae). All markers showed substantial polymorphism as well as revealing subtle genetic structures among the three genotyped populations. We also analyzed a fragment of the nuclear gene region of sodium/potassiumtransporting ATPase subunit alpha $(\mathrm{NaK})$ and a mitochondrial gene region partly coding for cytochrome oxidase subunit I ( $C O I)$ to demonstrate different genetic resolutions and sex-biased patterns of these markers, and their potential for combined use in future studies on the phylogeography and population genetics of $X$. concava. Although a limited number of populations was analyzed, we nevertheless obtained new insights on the latter two topics. The microsatellites revealed a generally high gene flow between the populations, but also suggested a deep historical segregation into two genetic lineages. This deep genetic segregation was confirmed by $\mathrm{NaK}$. While the high gene flow was unexpected, because of assumed restricted dispersal ability of $X$. concava and the discontinuous distribution of the host trees between the populations, the segregation of two lineages is comprehensible and could be explained by different refugial areas of the hosts during glacial times. The $\mathrm{COI}$ results showed a discordant strong genetic structure between all populations, which might be explained by the smaller effective population size of the mitochondrial genome. However, given the frequent evidence of a similar nature in recent studies on sawflies, we also consider and discuss mitochondrial introgression on population level as an alternative explanation.

\section{Introduction}

Xyelidae have always attracted the attention of taxonomists and systematists. They represent the sister group of the rest of the megadiverse insect order Hymenoptera (Ronquist et al., 2012; Klopfstein et al., 2013; Malm \& Nyman, 2015), which is traditionally divided into the paraphyletic "Symphyta" (missing the wasp waist) and the monophyletic Apocrita (sharing a wasp waist as a derived feature ) (Malm \& Nyman, 2015). The recent inconsistent phylogenetic placement of xyelids together with Pamphiliidae and Tenthredinidae as sister clade to all remaining Hymenoptera by Peters et al. (2017) might have been caused by an artificial grouping due to shared very slow mutation rates in those groups (Ronquist et al., 2012). The rich fossil record of Xyelidae includes the earliest fossil forms of Hymenoptera dating from the Middle-Upper Triassic (Kopylov, 2014). Proper knowledge of the phylogeography and 
population genetics of xyelids is therefore important in understanding the underlying evolutionary processes, which in turn will help to understand the evolution of other hymenopterans. Unfortunately, such data are scarce for xyelids due to the rarity of many species, ephemerality of the imagines, and considerable problems in identifying species morphologically as well as genetically (e.g., Burdick 1961; Blank, Shinohara \& Byun, 2005; Blank, Shinohara \& Altenhofer, 2013; Blank et al., 2017; Blank \& Kramp 2017). While a limited number of microsatellite studies has been conducted on sawflies (Hartel, Frederick \& Shanower, 2003; Cook et al., 2011; Caron et al., 2013; Bittner et al., 2017), none has focused on xyelids. Consequently, little is known about the population dynamics of this early-diverging group, including effects of ephemerality of imagines and their dispersal ability, host adaption and host dependency, and reproduction mode. Here, we report on the first developed and tested set of 16 polymorphic nuclear microsatellite markers for Xyela concava Burdick, 1961, to shed light on the latter issues. X. concava is widely distributed in southwestern USA, where it is closely associated with the pinyon-juniper woodland vegetation type of higher elevation semideserts, i.e., with pine species of the subgenus Strobus subsection Cembroides (Farjon 2010). Females oviposit into developing male cones of Pinus cembroides Zuccarini, 1830, P. edulis Engelmann, 1848 and P. monophylla Torrey \& Frémont, 1845 (Fig. 1), where the larvae feed on the sporophylls. After having ceased feeding, Xyela larvae dig into the soil below the host trees and may diapause up to several years before pupating (Blank, Shinohara \& Altenhofer, 2013). Imagines of the next generation emerge during spring and often visit flowering plants with easily accessible anthers, such as mountain mahogany (Cercocarpus spp.) and cliff-rose (Purshia spp.), from which they gather pollen for nutrition with their adapted mouthparts (Burdick 1961; Blank, personal observation). Flight behavior is described as erratic and slow (Burdick 1961). Therefore, given an assumed restricted dispersal ability and a close association with particular host species, it is intriguing to see how the variation within and among populations have been influenced by the distribution of the host trees during glacial and postglacial times. The high resolution power and therefore high capability of microsatellite markers to assess subtle and recent population genetic structures makes them well suited to this task. We used a commercial high-throughput sequencing approach for the development of the microsatellite markers and applied them to describe genetic structures and variation among and within three geographically distinct populations of $X$. concava. Furthermore, we compared the resolution of genetic variation of these markers with compiled data for one nuclear and one mitochondrial gene coding region and discuss their possible combined suitability for identifying genealogical lineages and answering phylogeographical questions. Finally, cross-amplification patterns for two species of Xyela and one of Pleroneura, sister taxon of Xyela (Smith 1967), are illustrated. 
91

92

93

94

95

96

97

\section{Material and methods}

\section{Sampling}

Xyela larvae were extracted from staminate cones of pines as described by Blank, Shinohara \& Altenhofer (2013) and stored in $100 \%$ ethanol at $-20{ }^{\circ} \mathrm{C}$. We included in the analysis larvae originating from three collection sites which are located 900-1,200 km from each other (see Table S1). The specimens are preserved in the Senckenberg Deutsches Entomologisches Institut, Müncheberg, Germany. Since it is impossible to identify Xyela larvae to species level morphologically, they were $C O I$ barcoded and identified by comparison with sequences from imagines identified as X. concava morphologically (identification following Burdick (1961), reference sequences of imagines were published by Blank, Kramp \& Shinohara (2017) and are deposited in the GenBank (NCBI) database, accession numbers KY198313 and KY198314). Finally, 98 larvae of $X$. concava were selected for the analysis (for detailed data see Table S1).

\section{DNA extraction}

Whole larvae were used for DNA extraction. The integument was slightly cut with a scalpel, so that the exterior stayed intact for later morphological inspection. DNA was extracted and purified with E.Z.N.A. Tissue DNA Kit (Omega Bio-Tek) according to the manufacturer's protocol, but with an extended 2 hour incubation time at $55^{\circ} \mathrm{C}$ (Thermomixer, without shaking) for cell lysis. The extracted DNA was stored at $-20^{\circ} \mathrm{C}$ until later use. The integuments were retained and stored in $70 \%$ ethanol.

\section{Microsatellite marker development and screening}

Total genomic DNA of a single female of $X$. concava (specimen ID: DEI-GISHym 30887, see Table S1) was extracted following the protocol described above. $10 \mathrm{ng} / \mu 1 \mathrm{DNA}$ in a total volume of $20 \mu 1$ was sent to AllGenetics \& Biology (Coruña, Spain) for the commercial identification of microsatellite motifs and the design of motif flanking primer pairs. A library was prepared for the DNA sample using the Nextera XTDNA kit (Illumina), following the manufacturer's instructions. The library was enriched with the following microsatellite motifs: AC, AG, ACG, and ATCT. Enriched DNA was sequenced in the Illumina MiSeq platform (PE300) and produced 3,043,190 paired-end reads. These paired-end reads were processed in Geneious 10.0.5 (Biomatters, Ltd.) using in-house developed scripts (property of AllGenetics \& Biology) and overlapped into 1,521,595 sequences (trim error probability limit of 0.03). Primer design was carried out by AllGenetics \& Biology in Primer 3 (Koressaar \& Remm 2007; Untergrasser et al., 2012) for 500 sequences containing microsatellite motifs. For a preliminary screening, fifty primer pairs were picked and four $X$. concava larvae (DEI-GISHym 32824-32827) were used for tests of polymorphism. Furthermore, 12 specimens of $X$. deserti Burdick, 1961, 12 specimens of an undescribed Xyela species, possibly a member of the X. alpigena group (Blank \& Kramp 2017), and six specimens of Pleroneura koebelei Rohwer, 1910 (see Table S1) were tested for cross-species amplification to check the marker system for potential use on two closely and one 
132

133

134

135

136

137

138

139

140

141

142

143

144

145

146

147

148

149

150

151

152

153

154

155

156

157

158

159

160

161

162

163

164

165

166

167

168

169

170

171

172

more distantly related xyelid species. The PCR analysis included a temperature gradient in the primer annealing step to find the best conditions for each primer pair. PCR was carried out in a total volume of $5 \mu 1$ containing $0.5 \mu 1$ DNA, $0.1 \mu$ of primers (10 pmol each) and $2.5 \mu 1$ of $2 \mathrm{x}$ Multiplex PCR Plus Master mix (QIAGEN). The PCR protocol consisted of an initial DNA polymerase (HotStar Taq) activation step at $95^{\circ} \mathrm{C}$ for $5 \mathrm{~min}$, followed by 35 cycles of $30 \mathrm{~s}$ of $95{ }^{\circ} \mathrm{C}$ (DNA denaturation step), $90 \mathrm{~s}$ at $50{ }^{\circ} \mathrm{C}, 52{ }^{\circ} \mathrm{C}, 54{ }^{\circ} \mathrm{C}$ and $56{ }^{\circ} \mathrm{C}$ (primer annealing step, temperature ramp), and $30 \mathrm{~s}$ at $72{ }^{\circ} \mathrm{C}$ (elongation step); the last cycle was followed by a final 10 min extension step at $68^{\circ} \mathrm{C} .5 \mu \mathrm{l}$ of the PCR product was visualized on a $2 \%$ agarose gel. Primer pairs that produced no amplification, multiple or unexpected size PCR products were discarded. Eighteen primer pairs, showing discernably strong and specific signals, were picked for further analysis. 5'-end fluorescently labelled reverse primers (6-Fam (Biomers) and NED, VIC, PET (Thermo Fisher Scientific)) for the selected primer pairs were synthesized for multiplexing and capillary electrophoresis. PCR was carried out in four multiplex reactions for four $X$. concava DNA samples in a total volume of $10 \mu$ l containing $2.5 \mu 1 \mathrm{DNA}, 1.0 \mu 1$ of fluorescently labelled primer pair mix $(0.5 \mathrm{pmol}$ each, containing up to five primer pairs, depending on compatible annealing temperature, dye and expected fragment size range) and $5.0 \mu 1$ of $2 x$ Multiplex PCR Plus Master mix (QIAGEN). PCR reaction conditions were as described above with the respective optimal annealing temperature for each primer pair mix. Reactions were diluted 1:2 and sent to Macrogen Europe (Amsterdam, the Netherlands) for fragment analysis.

Allele sizes were scored using GeneMapper 5.0 (Applied Biosystems). No marker showed strong stutter peaks or intensive background signal. Two primer pairs appeared to be monomorphic and were excluded from further analyses. Sixteen primer pairs showed apparent polymorphism for the four tested samples and were finally selected (Table 1).

\section{$\mathrm{COI}$ and $\mathrm{NaK}$ polymerase chain reaction analysis}

Primers used for amplification and sequencing are listed in Table 2. The mitochondrial region amplified is a $1,078 \mathrm{bp}$ long fragment of cytochrome oxidase subunit I gene (COI). The first $658 \mathrm{bp}$ of this fragment (from the 5 ' end) correspond to the standard barcode region of the animal kingdom (Hebert et al. 2004). Additionally, a 1,654 bp long fragment of the nuclear gene region of sodium/potassium-transporting ATPase subunit alpha $(\mathrm{NaK})$ was amplified.

PCR reactions were carried out in a total volume of 20-25 $\mu 1$ containing 1.5-3.0 $\mu 1$ DNA, 1.2$2.5 \mu 1$ of primers (5 pmol each) and 10.0-12.5 $\mu 1$ of 2x Multiplex PCR Plus Master mix (QIAGEN). The PCR protocol consisted of an initial DNA polymerase (HotStar Taq) activation step at $95{ }^{\circ} \mathrm{C}$ for $5 \mathrm{~min}$, followed by $38-40$ cycles of $30 \mathrm{~s}$ at $95^{\circ} \mathrm{C}, 90 \mathrm{~s}$ at $49-59{ }^{\circ} \mathrm{C}$ depending on the primer set used, and 50-120 s (depending on the amplicon size) at $72{ }^{\circ} \mathrm{C}$; the last cycle was followed by a final $30 \mathrm{~min}$ extension step at $68^{\circ} \mathrm{C} .3 \mu \mathrm{l}$ of the PCR product was visualized on a $1.4 \%$ agarose gel. Primers and dNTPs were inactivated with FastAP and Exonuclease I (Thermo Fisher Scientific). 1.7-2.2 U of both enzymes were added to 17-22 $\mu 1$ of PCR solution and incubated for $15 \mathrm{~min}$ at $37^{\circ} \mathrm{C}$, followed by $15 \mathrm{~min}$ at $85^{\circ} \mathrm{C}$. Purified PCR products were sent to Macrogen Europe (Amsterdam, the Netherlands) for sequencing. To obtain unequivocal 
173

174

175

176

177

178

179

180

181

182

183

184

185

186

187

188

189

190

191

192

193

194

195

196

197

198

199

200

201

202

203

204

205

206

207

208

209

210

sequences, both sense and antisense strands were sequenced. Sequences were aligned manually with Geneious 11.0.5. Ambiguous positions (i.e., double peaks in chromatograms of both strands) due to heterozygosity or heteroplasmy were coded using IUPAC symbols. Sequences have been deposited in the GenBank (NCBI) database (accession numbers MK265017MK265114 and MK264919-MK265016, for detailed data see Table S1).

\section{Genetic data analysis}

Estimations of genetic variation were obtained by calculating average number of alleles $\left(N_{\mathrm{A}}\right)$, observed $\left(H_{\mathrm{O}}\right)$ and expected heterozygosity $\left(H_{\mathrm{E}}\right)$ as well as deviations from Hardy-Weinberg equilibrium (HWE) for each locus for all $X$. concava populations using ARLEQUIN 3.5.2.2 (Excoffier \& Lischer, 2010) and 1,000 permutations. The same program was used to assess the suitability of resolving population differentiation by estimating population pairwise measures of $F_{\mathrm{ST}}(1,000$ permutations). The program GENEPOP 4.7.0 (Rousset, 2008) was used to estimate the inbreeding coefficient $F_{\text {IS }}$ (1,000 permutations). GENEPOP was also used in combination with the ENA correction implemented in the program FreeNA (Chapuis \& Estoup, 2007) to test for the presence and frequency of null alleles in the populations and to correct for the potential overestimation of $F_{\mathrm{ST}}$ values induced by the occurrence of null alleles (1,000 permutations). Number of genotypes (NG) in the dataset was counted with Excel. To test for isolation by distance, a Mantel test for the microsatellite data was performed (1,000 replicates) in ALLELES IN SPACE (Miller, 2005).

To assess the suitability of the microsatellite markers for assessing genetic population structures, three independent Bayesian assignment tests were carried out, one non-spatial using STRUCTURE 2.3.4 (Pritchard, Stephens \& Donnelly, 2000) and two spatial model based using BAPS 6.0 (Corander, Waldmann \& Sillanpää, 2003; Corander, Sirén \& Arjas, 2008) and GENELAND 4.0.8 (Guillot, Mortier \& Estoup, 2005). GENELAND assignment results for the microsatellite markers were also compared with results in GENELAND for the mitochondrial and nuclear gene coding markers (here without any comparison with a non-spatial assignment in STRUCTURE, since the model assumptions are likely to be violated for sequence data (Falush, Stephens \& Pritchard, 2003)). In BAPS, a maximum number of $10 \mathrm{~K}$ was given as a prior. In STRUCTURE, ten replicates for each $K$ from 1 to 10 were carried out with 50,000 burn-in steps followed by 100,000 MCMC. The online program STRUCTURE HARVESTER (Earl \& vonHoldt, 2012) was used to infer the most likely value of $K$. GENELAND was carried out with an uncertainty on coordinates of $25 \mathrm{~km}, 100,000$ iterations, a thinning to every 100 replicate and 10 independent runs. In STRUCTURE and GENELAND, a no admixture model and independency of allele frequency (uncorrelated model) was assumed, since correlated frequency models, though more powerful in detecting subtle differentiations, are more sensitive to departure from model assumptions (Guillot et al., 2012). 


\section{Results}

212 The identification of microsatellite motifs by using HTS yielded 500 potential markers of which

21350 were picked for a preliminary screening. Sixteen were finally implemented. Alongside primer

214 pairs that produced no amplification or were monomorphic, some also unexpectedly showed

215 PCR products of multiple sizes and had to be discarded.

216 The microsatellite markers amplified 3-14 different alleles and 3-18 different genotypes per

217 population and locus (Table 3). Observed heterozygosities ranged from 0.00 to 0.78 and were

218 significantly lower than those expected under Hardy-Weinberg equilibrium except for one locus,

219 indicating a deficiency of heterozygotes in the analyzed Xyela concava populations and/or the

220 presence of null alleles. This deficit is also confirmed by positive $F_{\text {IS }}$ values obtained for all but

221 three loci in one population. Estimated frequencies of null alleles were variable depending on the

222 respective microsatellite locus and $X$. concava population and varied between 0 and $39 \%$ (Table

223 4).

224 The estimation of the frequency of null alleles, though highly variable depending on the locuspopulation combination, did not introduce any bias to our dataset and thus did not cause an overestimation of pairwise $F_{\text {ST }}$ values.

The $F_{\mathrm{ST}}$ values uncorrected and corrected for the presence of null alleles showed higher values between the populations of Monitor Pass and Uinta Mountains as well as between the populations of Monitor Pass and Big Burro Mountains than the values between the populations of Uinta Mountains and Big Burro Mountains (Table 5). In general, all $F_{\mathrm{ST}}$ values were comparatively low (0.028-0.113) but either had a considerably narrow confidence interval or were significant or approaching the level of significance $(P=0.055)$. The $F_{\mathrm{ST}}$ values for $N a K$ and $C O I$ were, in comparison, higher (0.215-0.740). While the values for NaK showed the same pattern as the microsatellite markers in respect of genetic relationship of the populations, the $F_{\mathrm{ST}}$ values for $C O I$ indicated relatively high differences between all populations (Table 6). The Mantel test showed no isolation by distance $\left(r^{2}=0.0173, P<0.001\right)$. While spatial assignment tests for $\mathrm{NaK}$ and the mircosatellites came up with the same pattern as the $F_{\mathrm{ST}}$ values - as indicated by the assignment of two populations with high posterior probabilities to one genetic group or lineage (Figs. 2 and 3) - the non-spatial STRUCTURE analysis for microsatellites was slightly non-confirmative, with genotypes from the Big Burro Mountains and Uinta Mountains assigned to one separate lineage, yet with genotypes from all three populations assigned to one shared overlapping genetic lineage. The analysis of the $C O I$ data revealed that each population represented one distinct cluster $(K=3)$ (Fig. 3B, C, D). All microsatellite markers were successfully tested for cross-species amplification. For the three additional species of Xyela and Pleroneura, four markers showed polymorphic products and five were apparently monomorphic for $X$. deserti. Eight markers showed polymorphic products for the new Xyela species of the alpigena group, while no or unspecific fragments were amplified for Pleroneura koebelei (Table 7). 


\section{Discussion}

251 Of 50 initial markers, only 16 could finally be implemented. Such high drop-out rates due to

252

253

254

255

256

257

258

259

260

261

262

263

264

265

266

267

268

269

270

271

272

273

274

275

276

277

278

279

280

281

282

283

284

285

286

287

288

289 large numbers of repetitive motifs throughout the genome causing nonspecific binding of primers are already known (Schoebel et al., 2013). Other recent studies on invertebrates, using the same commercial HTS approach for the identification of SSR motifs, resulted in 11 to 21 polymorphic microsatellite markers, which nonetheless could be applied successfully (Reineke et al., 2015; González-Castellano et al., 2018; Gomes et al., 2019).

The analyses demonstrated that the degree of variability of the new microsatellite marker set is adequate in that it reveals polymorphic alleles within and across populations. The low significant deviations from Hardy-Weinberg equilibrium as well as positive $F_{\text {IS }}$ values for almost all loci in all populations could, however, have several causes. Given the ephemerality of the imagines and their fluctuating abundance due to extended diapausing, the major reason might have been a sampling bias, where only a fraction of each population was sampled (Wahlund effect). Furthermore, homozygote genotypes equally distributed across all populations indicated haploidy for altogether 26 specimens and may have had an impact on the discrepancy between the observed and expected heterozygosity. Thelytokous parthenogenesis - producing solely female offspring - which is known in xyelids (Blank, Shinohara \& Altenhofer, 2013), also might have contributed to the deficiency. However, due to the observed genotypic variation across the data set, apomictic parthenogenesis seems unlikely (Caron et al., 2013).

The results based on the non-spatial model in STRUCTURE were not as confirmative as in the spatial-model based assignment tests. Since in STRUCTURE no spatial information and therefore fewer assumptions are incorporated, geographical barriers and distance as likely causes for differentiated populations might have been underestimated (Coulon et al., 2006). On the other hand, because it does not include spatial information, STRUCTURE may here indicate a subtler genetic structure with possible higher exchange rates of the nuclear genome among all populations. However, both model applications told a broadly concordant narrative for the microsatellite markers, which are also supported by the low but significant $F_{\mathrm{ST}}$ values. First, the recent, seemingly discontinuous distribution of the hosts, $P$. edulis and P. monophylla, at higher elevations in mountain ranges with up to $100 \mathrm{~km}$ between single patches, apparently does not represent a barrier for recent and present gene flow. This is also supported by the Mantel test, which indicated no isolation by distance. $X$. concava is assumed to be relatively stationary due to the observed slow and erratic flight behavior (Burdick, 1961). Therefore, other explanations for the ability to disperse over long distances should be considered, such as passive dispersal by wind.

Second, the proposed geographically remote and restricted refugia of the host species during glacial times (Bentancourt et al., 1991; Grayson 2011; Duran, Pardo \& Mitton, 2012), and the considerably long distances between them, might have been sufficiently great to cause restricted gene flow and genetic segregation into two lineages. This assumption was also supported by the high and significant $F_{\mathrm{ST}}$ values and the genetic clustering of the NaK coding region, which due to the slower mutation rates presumably better reflects events in the past. In the $F_{\mathrm{ST}}$ statistics and

Peer) reviewing PDF | (2019:05:37530:2:0:NEW 1 Oct 2019) 
290

291

292

293

294

295

296

297

298

299

300

301

302

303

304

305

306

307

308

309

310

311

312

313

314

315

316

317

318

319

320

assignment tests of the microsatellite data (displaying presumably more recent events) the segregation could still be detected, but also a recent state of admixture was indicated. To test this hypothesis and a possibly ongoing admixture of the segregated lineages, populations of $X$. concava in hybrid zones and overlapping distribution areas of the host species should be included in future studies.

Compared to the results of the nuclear microsatellites and $N a K, F_{\mathrm{ST}}$ values and Bayesian statistics for the mitochondrial COI region showed a clear non-congruent pattern with a strong genetic structure among all three populations. One explanation could be the small effective population size $\left(N_{\mathrm{e}}\right)$ of the mitochondrial genome due to uniparental inheritance, which increases the rate at which populations will become genetically more structured (lineage sorting; Harrison, 1989). However, this non-congruent pattern also might have been caused by biased mitochondrial introgression as often found in "Symphyta" as recently discussed by Prous, Lee \& Mutanen (2019, preprint). The authors assume that mitochondrial introgression in sawflies might be promoted by a combination of the haplodiploid reproduction system of Hymenoptera and the low mitochondrial mutation rates in sawflies. The assumption is partly based on theoretical models of Patten, Carioscia \& Linnen (2015) showing that haplodiploid species are especially prone to biased mitochondrial introgression. Furthermore, Sloan, Havird \& Sharbrough (2017) recently suggested that species with low mitochondrial mutation rates might favor a specific beneficial (possibly locally adapted and/ or novel) mitochondrial haplotype to compensate for deleterious mitonuclear mutation loads. The specific haplotype then selectively sweeps through a population (or species) and purges deleterious mitochondrial mutations (the alternative solution being compensatory co-evolutionary changes in the nuclear genome). Tendentially, this would lead to a strong mitochondrial population structure and a mitonuclear discordance, which might be reflected in the data set. Given the evidence for the very low evolutionary rates of molecular characters in xyelids (Ronquist et al., 2012), this might be especially true for them. Additionally, mitochondrial introgression might likely be the cause for mitonuclear discordance in cases where there is a general agreement among large numbers of nuclear loci but discordance with mitochondrial genealogies (Sloan, Havird \& Sharbrough, 2017). Therefore, this new set of microsatellites may also be an attractive tool to indicate mitochondrial introgression at the population level of $X$. concava and other closely related xyelids. 


\section{Conclusions}

322 The implemented new set of microsatellite markers will be valuable for future analyses of

323 additional and less distantly located populations while unraveling the population structure of

324 Xyela concava. Together with other nuclear gene coding markers it can be used to elucidate both

325 old and recent divisions in the gene pool to reveal more details of the phylogeography of this

326 species. Furthermore, especially because of different underlying evolutionary processes affecting

327 the nuclear and mitochondrial genome, this new set of microsatellites can potentially be used to

328 reveal processes such as mitochondrial introgression at population level.

329 Even from this small data set, some tentative phylogeographic trends can be stated for $X$.

330 concava. This study covers only three populations but nevertheless indicates a segregation of two

331 genetic lineages and a recent state of admixture, which might have been caused by glacial retreat

332 events. This would agree with proposed geographically separate glacial refugia of the host

333 species. However, more populations covering the complete distribution of $X$. concava, especially

334 populations from overlapping distribution areas of the hosts, need to be analyzed to test this

335 hypothesis.

336 
337

338

339

340

341

342

343

344

345

346

347

348

349

350

351

352

353

354

355

356

357

358

359

360

361

362

363

364

365

366

367

368

369

370

371

372

373

374

375

376

\section{Acknowledgements}

We are grateful to C. Kutzscher (SDEI Müncheberg) for joining S.M. Blank during field work and for his support in the genetic lab. We thank A. Liston (SDEI Müncheberg) for a linguistic check of an earlier draft of the manuscript. We acknowledge the improvement of the manuscript by S.K. Monckton (Toronto) and an anonymous referee.

\section{References}

Aron S, de Menten L, Van Bockstaele DR, Blank SM, Roisin Y. 2005. When Hymenopteran Males Reinvented Diploidy. Current Biology 15:824-827.

Bittner TD, Hajek AE, Haavik L, Allison J, Nahrung H. 2017. Multiple introductions of Sirex noctilio (Hymenoptera: Siricidae) in northeastern North America based on microsatellite genotypes, and implications for biological control. Biological Invasions 19:1431-1447 DOI: $10.1007 / \mathrm{s} 10530-016-1365-1$.

Bentancourt JL, Schuster WS, Mitton JB, Anderson RS. 1991. Fossil and Genetic History of a Pinyon Pine (Pinus edulis). Ecology 72:1685-1697.

Blank SM, Kramp K. 2017. Xyela davidsmithi (Hymenoptera, Xyelidae), a new pine catkin sawfly with an unusual host association from the Sierra Nevada. Proceedings of the Washington Entomological Society 119:703-717.

Blank SM, Kramp K, Shinohara A. 2017. Xyela fusca spec. nov. from Japan elucidates East Asian-North American relationships of Xyela (Hymenoptera, Xyelidae). Zootaxa 4303:103-121 DOI: 10.11646/zootaxa.4303.1.6

Blank SM, Kramp K, Smith DR, Sundukov YN, Wei M, Shinohara A. 2017. Big and beautiful: The Megaxyela species (Hymenoptera, Xyelidae) of East Asia and North America. European Journal of Taxonomy 348:1-46 DOI: 10.5852/ejt.2017.348.

Blank SM, Shinohara A, Altenhofer E. 2013. The Eurasian species of Xyela (Hymenoptera, Xyelidae): Taxonomy, host plants and distribution. Zootaxa 3629:1-106 DOI: 10.11646/zootaxa.3629.1.1.

Blank SM, Shinohara A, Byun B-K. 2005. The East Asian Xyela species (Hymenoptera: Xyelidae) associated with Japanese Red Pine (Pinus densiflora; Pinaceae) and their distribution history. Insect Systematics \& Evolution 36:259-278 DOI: $10.1163 / 187631205788838393$.

Burdick DJ. 1961. A Taxonomic and Biological Study of the Genus Xyela Dalman in North America. University of California Publications in Entomology 17:285-356.

Caron V, Norgate M, Ede FJ, Nyman T, Sunnucks P. 2013. Novel microsatellite DNA markers indicate strict parthenogenesis and few genotypes in the invasive willow sawfly Nematus oligospilus. Bulletin of Entomological Research 103:74-88 DOI: 10.1017/S0007485312000429.

Chapuis MP, Estoup A. 2007. Microsatellite null alleles and estimation of population differentiation. Molecular Biology and Evolution 24:621-631 DOI: 10.1093/molbev/msl191. 
402

403

404

405

406

407

408

409

410

411

412

413

414

415

416

Cook N, Aziz N, Hedley PE, Morris J, Milne L, Karley AJ, Hubbard SF, Russell JR. 2011. Transcriptome sequencing of an ecologically important graminivorous sawfly: a resource for marker development. Conservation Genetics Resources 3:789-795 DOI: 10.1007/s12686-011-9459-7.

Corander J, Sirén J, Arjas E. 2008. Bayesian spatial modeling of genetic population structure. Computational Statistics 23:111-129 DOI: 10.1007/s00180-007-0072-x.

Corander J, Waldmann P, Sillanpää MJ. 2003. Bayesian analysis of genetic differentiation between populations. Genetics 163:367-374 DOI: 10.1093/bioinformatics/bth250.

Coulon A, Guillot G, Cosson J-F, Angibault JMA, Aulagnier S, Cargnelutti B, Galan M, Hewison AJM. 2006. Genetic structure is influenced by landscape features: empirical evidence from a roe deer population. Molecular Ecology 15:1669-1679 DOI: 10.1111/j.1365-294X.2006.02861.x.

Duran KL, Pardo A, Mitton JB. 2012. From middens to molecules: Phylogeography of the piñon pine, Pinus edulis. Journal of Biogeography 39:1536-1544 DOI: 10.1111/j.13652699.2012.02704.x.

Earl DA, vonHoldt BM. 2012. STRUCTURE HARVESTER: a website and program for visualizing STRUCTURE output and implementing the Evanno method. Conservation Genetics Resources 4:359-361 DOI: 10.1007/s12686-011-9548-7.

Excoffier L, Lischer HEL. 2010. An Integrated Software Package for Population Genetics Data Analysis. Molecular Ecology Resources 10:564-567 DOI: 10.1111/j.17550998.2010.02847.x.

Funk DJ, Omland KE. 2003. Species-Level Paraphyly and Polyphyly: Frequency, Causes, and Consequences, with Insights from Animal Mitochondrial DNA. Annual Review of Ecology, Evolution, and Systematics 34:397-423. DOI: 10.1146/annurev.ecolsys.34.011802.132421.

Gomes SO, Souza IGB, Santos MF, Silva GR, Albrecht M, McKinley AS, Bentzen P, Diniz FM. 2019. Discovery of novel NGS-mined microsatellite markers and an exploratory analysis of genetic differentiation between two Western Atlantic populations of Cardisoma guanhumi Latreille, 1825 (Decapoda: Brachyura: Gecarcinidae). Journal of Crustacean Biology 39:181-185. DOI: 10.1093/jcbiol/ruy115.

González-Castellano I, Perina A, González-Tizón AM, Torrecilla Z, Martínez-Lage A. 2018. Isolation and characterization of 21 polymorphic microsatellite loci for the rockpool shrimp Palaemon elegans using Illumina MiSeq sequencing. Scientific Reports 8:8-13. DOI: 10.1038/s41598-018-35408-1.

Grayson DK. 2011. The Great Basin: A Natural Prehistory. Berkley and Los Angeles: University of California Press.

Guillot G, Mortier F, Estoup A. 2005. GENELAND: A computer package for landscape genetics. Molecular Ecology Notes 5:712-715 DOI: 10.1111/j.1471-8286.2005.01031.x.

Guillot G, Renaud S, Ledevin R, Michaux J, Claude J. 2012. A Unifying Model for the Analysis of Phenotypic, Genetic, and Geographic Data. Systematic Biology 61:897-911 DOI: 10.1093/sysbio/sys038. 
417

418

419

420

421

422

423

424

425

426

427

428

429

430

431

432

433

434

435

436

437

438

439

440

441

442

443

444

445

446

447

448

449

450

451

452

453

454

455

456

Harrison RG. 1989. Animal mitochondrial DNA as a genetic marker in population and evolutionary biology. Trends in Ecology and Evolution 4:6-11. DOI: 10.1016/01695347(89)90006-2.

Hartel KD, Frederick BA, Shanower TG. 2003. Isolation and characterization of microsatellite loci in wheat stem sawfly Cephus cinctus and cross-species amplification in related species. Molecular Ecology Notes 3:85-87 DOI: 10.1046/j.1471-8286.

Hebert PDN, Penton EH, Burns JM, Janzen DH, Hallwachs W. 2004. Ten species in one: DNA barcoding reveals cryptic species in the neotropical skipper butterfly Astraptes fulgerator. Proceedings of the National Academy of Sciences of the United States of America 101:14812-14817. DOI: 10.1073/pnas.0406166101.

Klopfstein S, Vilhelmsen L, Heraty JM, Sharkey M, Ronquist F. 2013. The Hymenopteran Tree of Life: Evidence from Protein-Coding Genes and Objectively Aligned Ribosomal Data. PLoS ONE 8. DOI: 10.1371/journal.pone.0069344.

Kopylov DS. 2014. New sawflies of the subfamily Madygellinae (Hymenoptera, Xyelidae) from the Middle-Upper Triassic of Kyrgyzstan. Paleontological Journal 48:610-620 DOI: 10.1134/s0031030114060070.

Koressaar T, Remm M. 2007. Enhancements and modifications of primer design program Primer3. Bioinformatics 23:1289-1291. DOI: 10.1093/bioinformatics/btm091.

Malm T, Nyman T. 2015. Phylogeny of the symphytan grade of Hymenoptera: new pieces into the old jigsaw(fly) puzzle. Cladistics 31:1-17. DOI: 10.1111/cla.12069.

Miller MP. 2005. Alleles In Space (AIS): Computer software for the joint analysis of interindividual spatial and genetic information. Journal of Heredity 96:722-724. DOI: 10.1093/jhered/esi119.

Patten MM, Carioscia SA, Linnen CR. 2015. Biased introgression of mitochondrial and nuclear genes: A comparison of diploid and haplodiploid systems. Molecular Ecology 24:5200 5210. DOI: $10.1111 / \mathrm{mec} .13318$.

Peters RS, Krogmann L, Mayer C, Donath A, Gunkel S, Meusemann K, Kozlov A, Podsiadlowski L, Petersen M, Lanfear R, Diez PA, Heraty J, Kjer KM, Klopfstein S, Meier R, Polidori C, Schmitt T, Liu S, Zhou X, Wappler T, Rust J, Misof B, Niehuis O. 2017. Evolutionary History of the Hymenoptera. Current Biology 27:1013-1018. DOI: 10.1016/j.cub.2017.01.027.

Pritchard JK, Stephens M, Donnelly P. 2000. Inference of population structure using multilocus genotype data. Genetics 155:945-959 DOI: 10.1111/j.1471-8286.2007.01758.x.

Prous M, Kramp K, Vikberg V, Liston A. 2017. North-Western Palaearctic species of Pristiphora (Hymenoptera, Tenthredinidae). Journal of Hymenoptera Research 59:1-190 DOI: $10.3897 / \mathrm{jhr} .59 .12656$.

Prous M, Lee KM, Mutanen M. 2019. Detection of cross-contamination and strong mitonuclear discordance in two species groups of sawfly genus Empria. bioRxiv. DOI: http://dx.doi.org/10.1101/525626.

Prous M, Vikberg V, Liston A, Kramp K. 2016. North-Western Palaearctic species of the 
457

458

459

460

461

462

463

464

465

466

467

468

469

470

471

472

473

474

475

476

477

478

479

480

481

482

483

Pristiphora ruficornis group (Hymenoptera, Tenthredinidae). Journal of Hymenoptera Research 51:1-54 DOI: 10.3897/jhr.51.9162.

Reineke A, Assaf HA, Kulanek D, Mori N, Pozzebon A, Duso C. 2015. A novel set of microsatellite markers for the European Grapevine Moth Lobesia botrana isolated using next-generation sequencing and their utility for genetic characterization of populations from Europe and the Middle East. Bulletin of Entomological Research 105:408-416. DOI: 10.1017/S0007485315000267.

Ronquist F, Klopfstein S, Vilhelmsen L, Schulmeister S, Murray DL, Rasnitsyn AP. 2012. A Total-Evidence Approach to Dating with Fossils, Applied to the Early Radiation of the Hymenoptera. Systematic Biology 61:973-999 DOI: 10.1093/sysbio/sys058.

Rousset F. 2008. GENEPOP'007: A complete re-implementation of the GENEPOP software for Windows and Linux. Molecular Ecology Resources 8:103-106 DOI: 10.1111/j.14718286.2007.01931.x.

Schoebel CN, Brodbeck S, Buehler D, Cornejo C, Gajurel J, Hartikainen H, Keller D, Leys M, Ř́ćčanová Š, Segelbacher G, Werth S, Csencsics D. 2013. Lessons learned from microsatellite development for nonmodel organisms using 454 pyrosequencing. Journal of Evolutionary Biology 26:600-611. DOI: 10.1111/jeb.12077.

Sloan DB, Havird JC, Sharbrough J. 2017. The on-again, off-again relationship between mitochondrial genomes and species boundaries. Molecular Ecology 26:2212-2236. DOI: $10.1111 / \mathrm{mec} .13959$.

Smith DR. 1967. A review of the larvae of Xyelidae, with notes on the family classification (Hymenoptera). Annals of the Entomological Society of America 60:376-384. DOI: 10.1093/aesa/60.2.376.

Untergasser A, Cutcutache I, Koressaar T, Ye J, Faircloth BC, Remm M, Rozen SG. 2012. Primer3 - new capabilities and interfaces. Nucleic Acids Research 40:1-12. DOI: 10.1093/nar/gks596. 


\section{Table $\mathbf{1}$ (on next page)}

Sixteen polymorphic microsatellite loci and the corresponding flanking primer pairs identified in the pine catkin sawfly Xyela concava 


\begin{tabular}{|c|c|c|c|c|c|}
\hline Locus & $\begin{array}{l}\text { Size range } \\
\text { (bp) }\end{array}$ & Motif & $\begin{array}{c}\mathrm{Ta} \text { in } \\
{ }^{\circ} \mathrm{C}\end{array}$ & label & Primer sequence $\left(5^{\prime}-3^{\prime}\right)$ \\
\hline AG_30887_445 & $75-93$ & $\mathrm{AAG}_{(11)}$ & 50 & VIC & $\begin{array}{l}\text { F: GTCTCGACTCCCTCCTACGA } \\
\text { R: ACGGAAGTGCATCGGATCTTC }\end{array}$ \\
\hline AG_30887_046 & $195-225$ & $\mathrm{AGC}_{(30)}$ & 50 & PET & $\begin{array}{l}\text { F: CCTTTCGTCCTGGTTGACCA } \\
\text { R: GATACGCCAGCCTATCCGTC }\end{array}$ \\
\hline AG_30887_083 & $178-190$ & $\mathrm{AAG}_{(10)}$ & 50 & 6-Fam & $\begin{array}{l}\text { F: TTCCAGTTTCTTGCAACGCG } \\
\text { R: ATTCGCAAGCCTCTTCTGCA }\end{array}$ \\
\hline AG_30887_188 & $179-188$ & $\operatorname{AAT}_{(9)}$ & 50 & NED & $\begin{array}{l}\text { F: GCGGCGGTATAATGAGTCGT } \\
\text { R: GGAAAGTGACTGCTACCGGT }\end{array}$ \\
\hline AG_30887_479 & $93-102$ & $\mathrm{ACT}_{(8)}$ & 50 & PET & $\begin{array}{l}\text { F: GCTGTTCACATGGCAGGTAG } \\
\text { R: CCACCATCCCTACTACGGCT }\end{array}$ \\
\hline AG_30887_193 & $110-134$ & $\mathrm{AGC}_{(17)}$ & 50 & VIC & $\begin{array}{l}\text { F: AGAGTGCCAACGTGGGAAAT } \\
\text { R: TTACTTTGCCCATGCCATGC }\end{array}$ \\
\hline AG_30887_234 & $376-424$ & $\operatorname{AATGCG}_{(8)}$ & 50 & PET & $\begin{array}{l}\text { F: AGTCTGATCCTTCCTGCGGA } \\
\text { R: ATACGTGCCAGTTCGATCGT }\end{array}$ \\
\hline AG_30887_282 & $239-263$ & $\mathrm{AGC}_{(10)}$ & 50 & 6-Fam & $\begin{array}{l}\text { F: CTGTGCCTACGTCCCTTAGG } \\
\text { R: CCCATCGTTTGGTCGGTAGA }\end{array}$ \\
\hline AG_30887_286 & $103-121$ & $\mathrm{AGC}_{(8)}$ & 50 & NED & $\begin{array}{l}\text { F: GCGTCCGTCTGAAATCTTGG } \\
\text { R: CATTCGCATTCGACGCACTC }\end{array}$ \\
\hline AG_30887_179 & $111-126$ & $\mathrm{AGC}_{(9)}$ & 50 & 6-Fam & $\begin{array}{l}\text { F: CCCGTTCGTAAATCGGTCCT } \\
\text { R: GACGTGGAATCGGTGGACTC }\end{array}$ \\
\hline $\mathrm{AG} \_30887 \_460$ & $90-116$ & $\mathrm{AT}_{(5)}$ & 50 & PET & $\begin{array}{l}\text { F: ACGTACTTATTGGGCGCGAA } \\
\text { R: TTTACATGCTGTACACCGGGA }\end{array}$ \\
\hline AG_30887_347 & $237-249$ & $\mathrm{AAG}_{(8)}$ & 50 & PET & $\begin{array}{l}\text { F: CCCGGACCTCGTGCTATTC } \\
\text { R: GGCGACAATCCCACGTGATA }\end{array}$ \\
\hline$A G \_30887 \_393$ & $136-175$ & $\mathrm{AAG}_{(8)}$ & 50 & 6-Fam & $\begin{array}{l}\text { F: CCATCACTGTGCCGCGATAT } \\
\text { R: GCACCTCAGGGATCCTCAAT }\end{array}$ \\
\hline AG_30887_414 & $122-179$ & $\mathrm{AAG}_{(8)}$ & 50 & NED & $\begin{array}{l}\text { F: TGATTTGTGCAACCGAGGGA } \\
\text { R: CCCTTTATTCTCAGCAACCGC }\end{array}$ \\
\hline AG_30887_012 & $130-148$ & $\mathrm{AGG}_{(9)}$ & 50 & PET & $\begin{array}{l}\text { F: TTCCGGACGACTTTGACCTG } \\
\text { R: CCTCGATTCCGATTCCCGTT }\end{array}$ \\
\hline $\mathrm{AG} \_30887 \_223$ & $120-186$ & $\mathrm{AAG}_{(9)}$ & 50 & 6-Fam & $\begin{array}{l}\text { F: TCAAAGCGGAGAAAGAGCGT } \\
\text { R: TTAACCGCCATCGACCGTTC }\end{array}$ \\
\hline
\end{tabular}




\section{Table 2 (on next page)}

Nuclear NaK and mitochondrial COI primers used for amplification (PCR) and sequencing (seq) 


\begin{tabular}{lccccc} 
Gene Region & Primer name & Primer sequence (5'-3') & Ta in ${ }^{\circ} \mathrm{C}$ & PCR/ Sequencing & Reference \\
\hline COI & symF1 & TTTCAACWAATCATAAARAYATTGG & 49 & PCR, seq & Prous et al. 2016 \\
COI & symR1 & TAAACTTCWGGRTGICCAAARAATC & 49 & PCR/ seq & Prous et al. 2016 \\
COI & symC1-J1751 & GGAGCNCCTGATATAGCWTTYCC & 49 & seq & Prous et al. 2016 \\
NaK & NaK263F & CTYAGCCAYGCRAARGCRAARGA & 59 & PCR/ seq & Prous et al. 2017 \\
NaK & NaK907Ri & TGRATRAARTGRTGRATYTCYTTIGC & 59 & seq & Prous et al. 2017 \\
NaK & NaK1250Fi & ATGTGGTTYGAYAAYCARATYATIGA & 59 & seq & Prous et al. 2017 \\
NaK & NaK1918R & GATTTGGCAATNGCTTTGGCAGTDAT & 59 & PCR/ seq & Prous et al. 2017
\end{tabular}




\section{Table 3 (on next page)}

Comparative genetic diversity values for the three Xyela concava populations

Analyzed for each of the 16 microsatellite loci and on average over all loci including number of alleles $\left(N_{A}\right)$, Number of genotypes $(N G)$, observed $\left(H_{0}\right)$ and expected $\left(H_{\mathrm{E}}\right)$ heterozygosity and estimates of $F_{15}$ 


\begin{tabular}{|c|c|c|c|c|c|c|c|c|c|c|c|c|}
\hline \multirow{2}{*}{ Locus } & \multicolumn{4}{|c|}{ Big Burro Mountains } & \multicolumn{4}{|c|}{ Monitor Pass } & \multicolumn{4}{|c|}{ Uinta Mountains } \\
\hline & $N_{\mathrm{A}} / \mathrm{NG}$ & $F_{\text {IS }}$ & $H_{\mathrm{O}}$ & $H_{\mathrm{E}}$ & $N_{\mathrm{A}} / \mathrm{NG}$ & $F_{\text {IS }}$ & $H_{\mathrm{O}}$ & $H_{\mathrm{E}}$ & $N_{\mathrm{A}} / \mathrm{NG}$ & $F_{\text {IS }}$ & $H_{\mathrm{O}}$ & $H_{\mathrm{E}}$ \\
\hline AG_30887_445 & $6 / 7$ & 0.91 & 0.07 & $0.78 *$ & $6 / 11$ & 0.50 & 0.40 & $0.79 *$ & $7 / 12$ & 0.43 & 0.40 & $0.69 *$ \\
\hline AG_30887_046 & $10 / 11$ & 0.33 & 0.61 & $0.85^{*}$ & $9 / 14$ & 0.44 & 0.47 & $0.82 *$ & $7 / 11$ & 0.04 & 0.78 & $0.80 *$ \\
\hline AG_30887_083 & $5 / 7$ & 0.39 & 0.43 & $0.69 *$ & $3 / 5$ & 0.63 & 0.20 & $0.53 *$ & $4 / 5$ & -0.18 & 0.63 & $0.53 *$ \\
\hline AG_30887_188 & $4 / 6$ & 0.84 & 0.11 & $0.66^{*}$ & $3 / 5$ & 0.31 & 0.37 & $0.53 *$ & $3 / 5$ & 0.63 & 0.25 & $0.66^{*}$ \\
\hline AG_30887_479 & $3 / 4$ & 0.22 & 0.43 & $0.54^{*}$ & $4 / 5$ & 0.79 & 0.10 & $0.47^{*}$ & $4 / 6$ & -0.06 & 0.53 & $0.49 *$ \\
\hline AG_30887_193 & $6 / 8$ & 0.31 & 0.50 & $0.72 *$ & $5 / 12$ & 0.47 & 0.40 & $0.74^{*}$ & $7 / 13$ & 0.04 & 0.78 & $0.80 *$ \\
\hline AG_30887_234 & $6 / 9$ & 0.34 & 0.50 & $0.75^{*}$ & $6 / 8$ & 0.42 & 0.40 & $0.68 *$ & $6 / 9$ & 0.21 & 0.63 & $0.78 *$ \\
\hline AG_30887_282 & $8 / 8$ & 0.40 & 0.46 & $0.77^{*}$ & $6 / 9$ & 0.61 & 0.30 & $0.75^{*}$ & $6 / 9$ & -0.03 & 0.73 & $0.70 *$ \\
\hline AG_30887_286 & $6 / 8$ & 0.76 & 0.18 & $0.74 *$ & $5 / 9$ & 0.24 & 0.47 & 0.61 & $7 / 11$ & 0.53 & 0.35 & $0.74 *$ \\
\hline AG_30887_179 & $3 / 3$ & 1.00 & 0.00 & $0.62 *$ & $5 / 7$ & 0.55 & 0.20 & $0.43 *$ & $5 / 6$ & 0.76 & 0.15 & $0.61 *$ \\
\hline AG_30887_460 & $6 / 6$ & 0.75 & 0.14 & $0.55^{*}$ & $4 / 4$ & 0.30 & 0.13 & $0.18^{*}$ & $6 / 6$ & 0.74 & 0.15 & $0.56 *$ \\
\hline AG_30887_347 & $4 / 5$ & 0.34 & 0.43 & $0.64^{*}$ & $3 / 6$ & 0.51 & 0.33 & $0.67^{*}$ & $4 / 7$ & 0.06 & 0.63 & $0.66 *$ \\
\hline AG_30887_393 & $7 / 7$ & 0.82 & 0.11 & $0.59 *$ & $6 / 10$ & 0.44 & 0.40 & $0.71 *$ & $5 / 9$ & 0.67 & 0.20 & $0.59 *$ \\
\hline AG_30887_414 & $12 / 12$ & 0.35 & 0.54 & $0.82 *$ & $10 / 18$ & 0.54 & 0.40 & $0.86^{*}$ & $9 / 13$ & 0.13 & 0.68 & $0.77^{*}$ \\
\hline AG_30887_012 & $5 / 7$ & 0.90 & 0.07 & $0.73 *$ & $3 / 4$ & 0.51 & 0.27 & $0.54 *$ & $3 / 4$ & 0.67 & 0.23 & $0.67 *$ \\
\hline AG_30887_223 & $9 / 11$ & 0.76 & 0.14 & $0.80 *$ & $14 / 18$ & 0.36 & 0.47 & $0.89 *$ & $13 / 15$ & 0.72 & 0.23 & $0.82 *$ \\
\hline Mean & & 0.59 & 0.29 & 0.71 & & 0.48 & 0.33 & 0.64 & & 0.33 & 0.46 & 0.68 \\
\hline S.D. & & 0.26 & 0.21 & 0.09 & & 0.13 & 0.12 & 0.18 & & 0.33 & 0.24 & 0.10 \\
\hline
\end{tabular}




\section{Table 4 (on next page)}

Estimated null allele frequencies for each of the 16 polymorphic microsatellite loci and each population including the average null allele frequency 
Estimated null allele frequency

\begin{tabular}{llll}
\hline Locus & Big Burro Mts & Monitor Pass & Uinta Mts \\
\hline AG_30887_445 & 0.395 & 0.221 & 0.167 \\
AG_30887_046 & 0.165 & 0.191 & 0.028 \\
AG_30887_083 & 0.175 & 0.229 & 0.041 \\
AG_30887_188 & 0.334 & 0.116 & 0.247 \\
AG_30887_479 & 0.095 & 0.267 & 0.040 \\
AG_30887_193 & 0.130 & 0.194 & 0.037 \\
AG_30887_234 & 0.161 & 0.184 & 0.087 \\
AG_30887_282 & 0.194 & 0.260 & 0.036 \\
AG_30887_286 & 0.314 & 0.073 & 0.208 \\
AG_30887_179 & 0.381 & 0.190 & 0.282 \\
AG_30887_460 & 0.259 & 0.000 & 0.257 \\
AG_30887_347 & 0.148 & 0.200 & 0.048 \\
AG_30887_393 & 0.309 & 0.163 & 0.247 \\
AG_30887_414 & 0.196 & 0.245 & 0.053 \\
AG_30887_012 & 0.378 & 0.183 & 0.264 \\
AG_30887_223 & 0.319 & 0.162 & 0.314 \\
\hline Mean & 0.247 & 0.180 & 0.147
\end{tabular}




\section{Table 5 (on next page)}

Pairwise $F_{\mathrm{ST}}$ estimates between populations of Xyela concava for the 16 microsatellite loci including corresponding $P$ values and confidence intervals

Estimates are given both uncorrected and corrected for the presence of null alleles. Bold typeface denotes pairwise $F_{\mathrm{ST}}$ estimates that are significantly different from zero $(P<0.005)$. Values in square brackets indicate $95 \%$ confidence intervals for pairwise corrected $F_{\text {ST }}$ estimates 


\begin{tabular}{lccc}
$F_{\mathrm{ST}}$ uncorrected & Big Burro Mts & Monitor Pass & Uinta Mts \\
\hline Big Burro Mts & $*$ & $*$ & \\
Monitor Pass & $\mathbf{0 . 0 9 1 8 2}$ & $\mathbf{0 . 0 7 7 0 5}$ & $*$ \\
Uinta Mts & 0.02254 & Monitor Pass & Uinta Mts \\
\hline$F_{\mathrm{ST}}$ ENA corrected & Big Burro Mts & & \\
Big Burro Mts & $*$ & $*$ & $*$ \\
Monitor Pass & $0.083[0.054,0.115]$ & & \\
Uinta Mts & $0.015[0.004,0.028]$ & $0.065[0.041,0.094]$ & \\
\hline
\end{tabular}

1 


\section{Table 6(on next page)}

Pairwise $F_{\mathrm{ST}}$ estimates between populations of Xyela concava for $\mathrm{NaK}$ and $\mathrm{CO}$ including corresponding $P$ values

Bold typeface denotes pairwise $F_{S T}$ estimates that are significantly different from zero $(P<$ $0.005)$ 


\begin{tabular}{lccc}
$\mathbf{N a} \boldsymbol{K}$ & BB Mts & Mon Pass & Uinta Mts \\
\hline Big Burro & $*$ & & \\
Mts & & & \\
Monitor Pass & $\mathbf{0 . 7 4 0}$ & $*$ & \\
Uinta Mts & $\mathbf{0 . 2 1 5}$ & $\mathbf{0 . 6 8 0}$ & $*$ \\
\hline COI & BB Mts & Mon Pass & Uinta Mts \\
\hline Big Burro & $*$ & & \\
Mts & & & \\
Monitor Pass & $\mathbf{0 . 6 9 9}$ & $*$ & \\
Uinta Mts & $\mathbf{0 . 5 0 8}$ & $\mathbf{0 . 6 7 8}$ & $*$
\end{tabular}


Table 7 (on next page)

Cross-species amplification

(-) no product, $(+)$ monomorphic product, $(++)$ polymorphic product 


\begin{tabular}{|c|c|c|c|}
\hline Locus & Xyela deserti & Xyela spec. nov. & Pleroneura koebelei \\
\hline $\mathrm{AG} \_30887 \_445$ & - & - & - \\
\hline AG_30887_046 & + & ++ & - \\
\hline AG_30887_083 & - & - & - \\
\hline $\mathrm{AG} \_30887 \_188$ & - & - & - \\
\hline $\mathrm{AG} \_30887 \_479$ & + & ++ & - \\
\hline AG_30887_193 & - & ++ & - \\
\hline AG_30887_234 & + & ++ & - \\
\hline AG_30887_282 & ++ & ++ & - \\
\hline $\mathrm{AG}$ _30887_286 & ++ & ++ & - \\
\hline $\mathrm{AG} \_30887 \_179$ & - & - & - \\
\hline $\mathrm{AG} \_30887 \_460$ & - & - & - \\
\hline $\mathrm{AG} \_30887 \_347$ & ++ & ++ & - \\
\hline AG_30887_393 & + & - & - \\
\hline $\mathrm{AG} \_30887 \_414$ & ++ & - & - \\
\hline AG_30887_012 & - & - & - \\
\hline AG_30887_223 & + & ++ & - \\
\hline
\end{tabular}


Figure 1

Location of the collection areas and distribution of the host species

[p]Credit Pinus spec. shape files: https://data.usgs.gov/metadata[p]

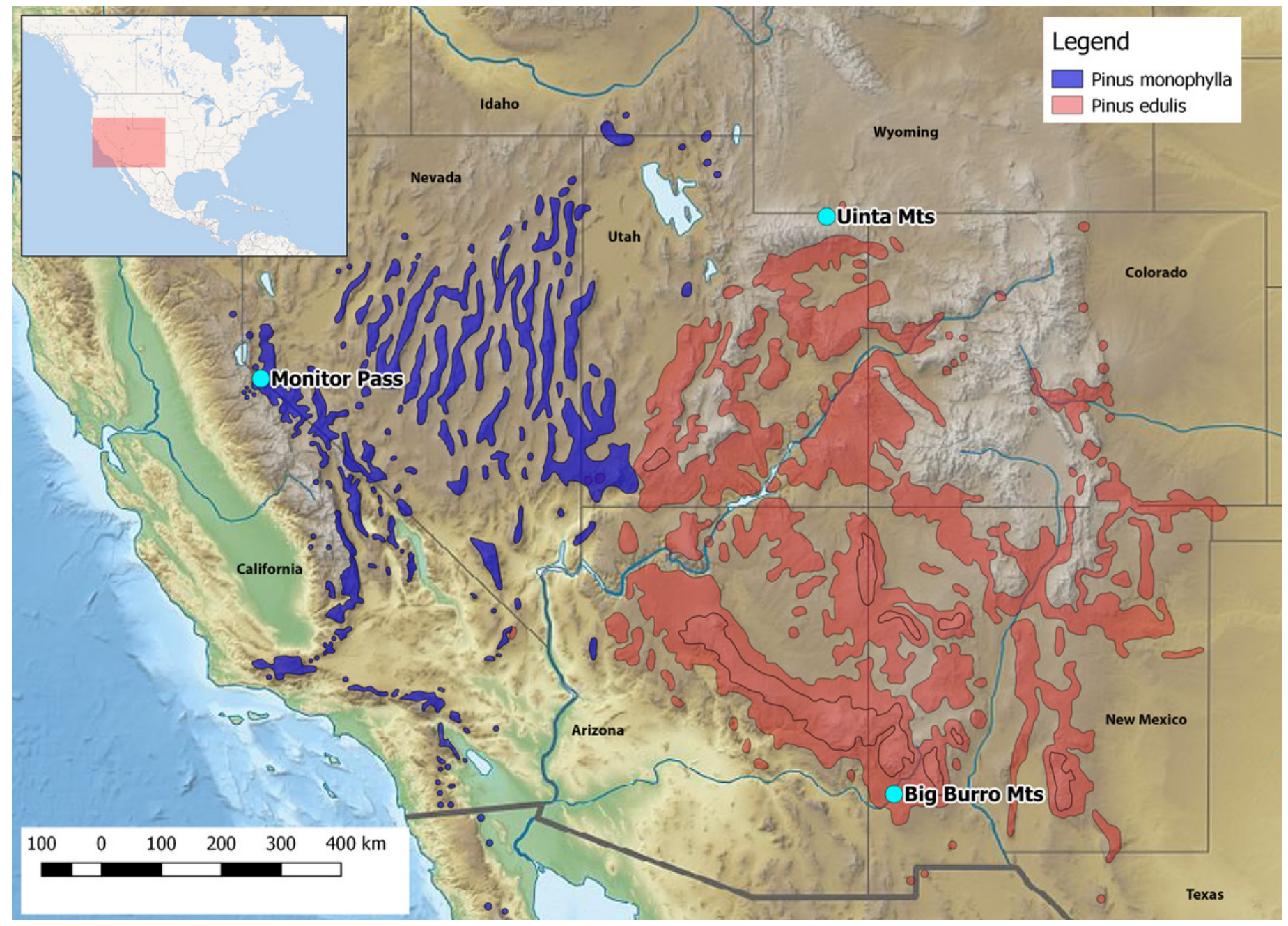




\section{Figure 2}

Bayesian assignment of Xyela concava populations to each of the identified clusters ( $K$ $=2$ ) for the microsatellite markers

(A) GENELAND (Posterior probabilities are indicated in the scale bar. The contour lines in the maps indicate the spatial positions of genetic discontinuities. Lighter shading indicates a higher probability of belonging to the genetic population), (B) BAPS (the area of each population is proportional to the number of specimens used) and (C) STRUCTURE

A

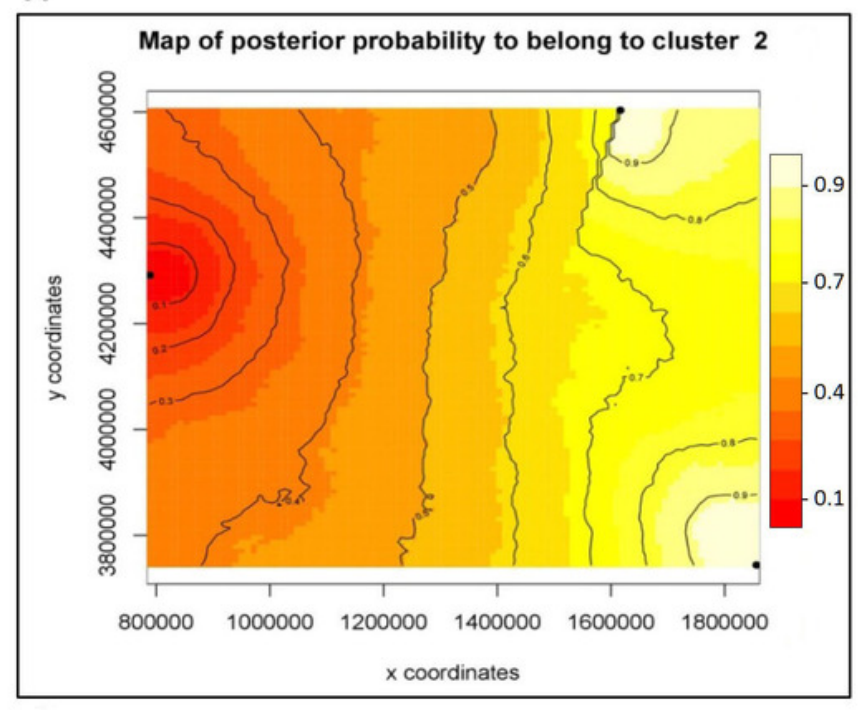

C
B

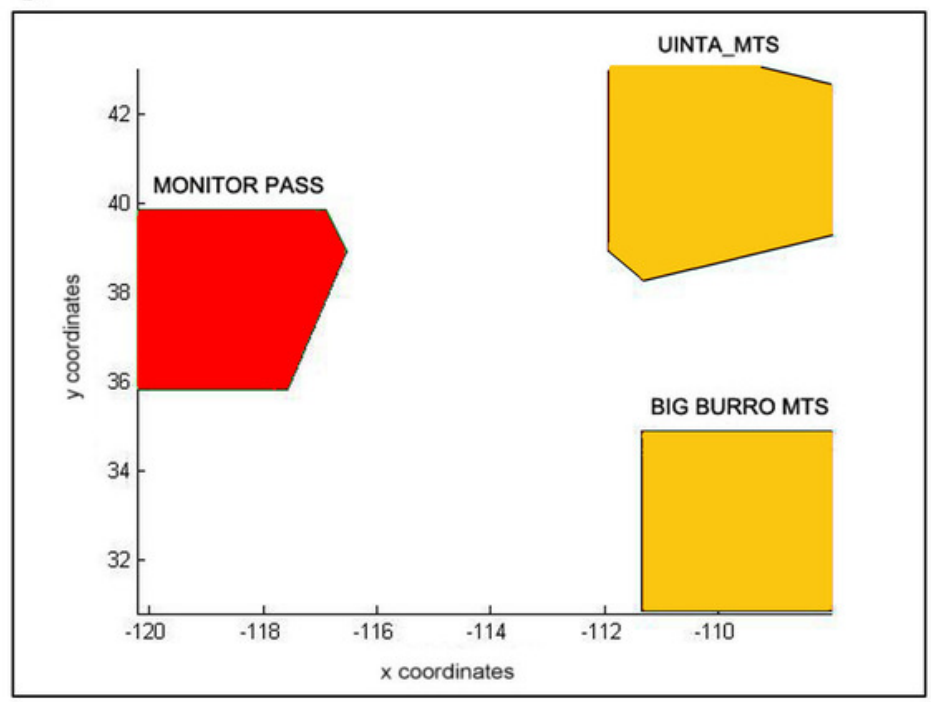

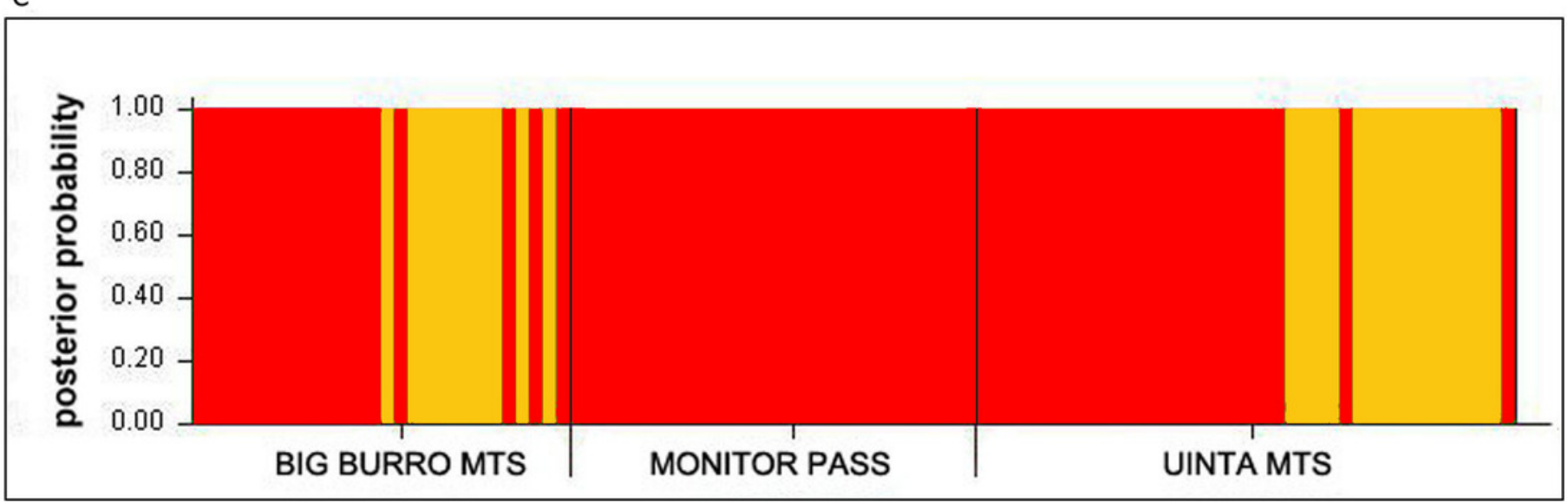




\section{Figure 3}

Bayesian spatial assignment (GENELAND) of Xyela concava populations to each of the identified clusters for (A) NaK $(K=2)$ and (B), (C), (D) COI $(K=3)$.

The different colors represent the estimated posterior probabilities of the membership to each cluster. Posterior probabilities are indicated in the scale bar. The contour lines in the maps indicate the spatial positions of genetic discontinuities. Lighter shading indicates a higher probability of belonging to the genetic population. 
A

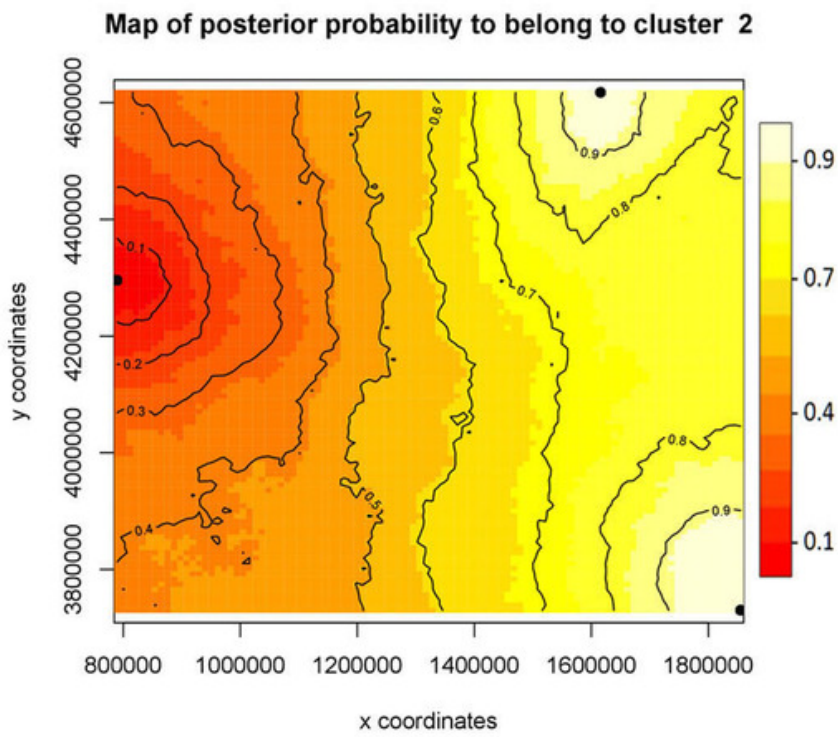

C

Map of posterior probability to belong to cluster 2

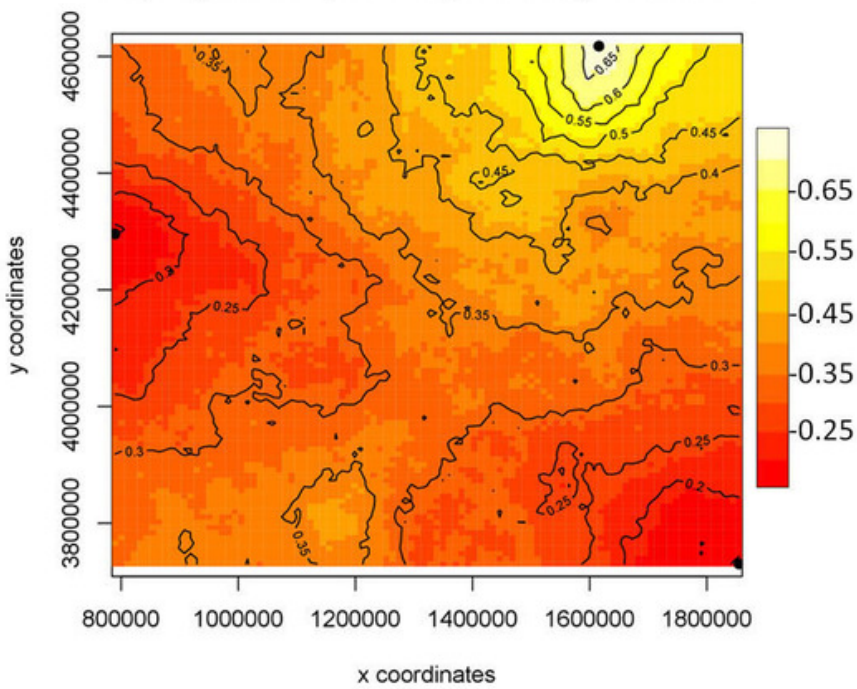

B

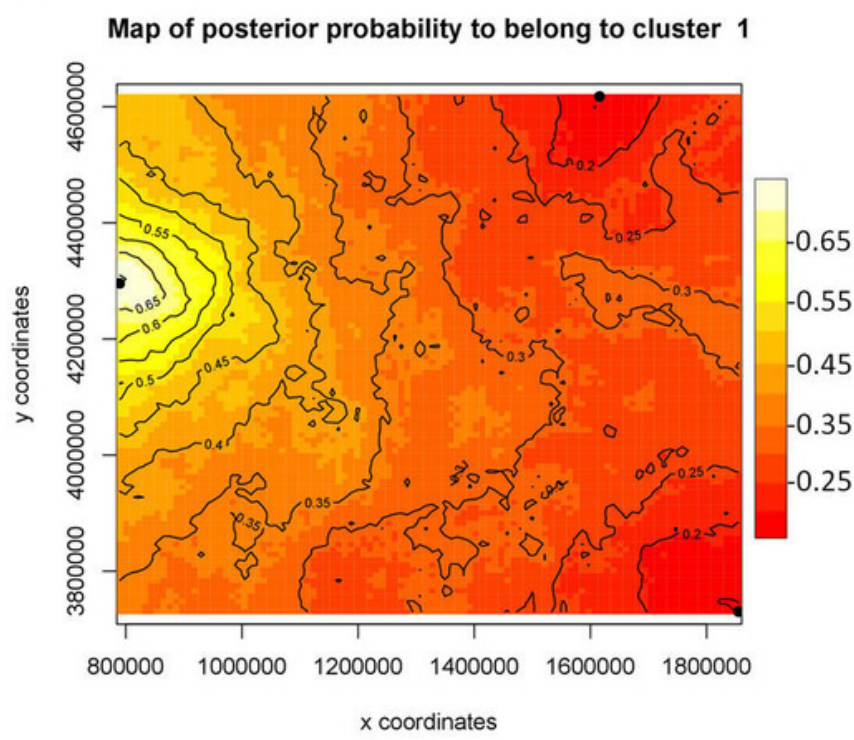

D

Map of posterior probability to belong to cluster 3

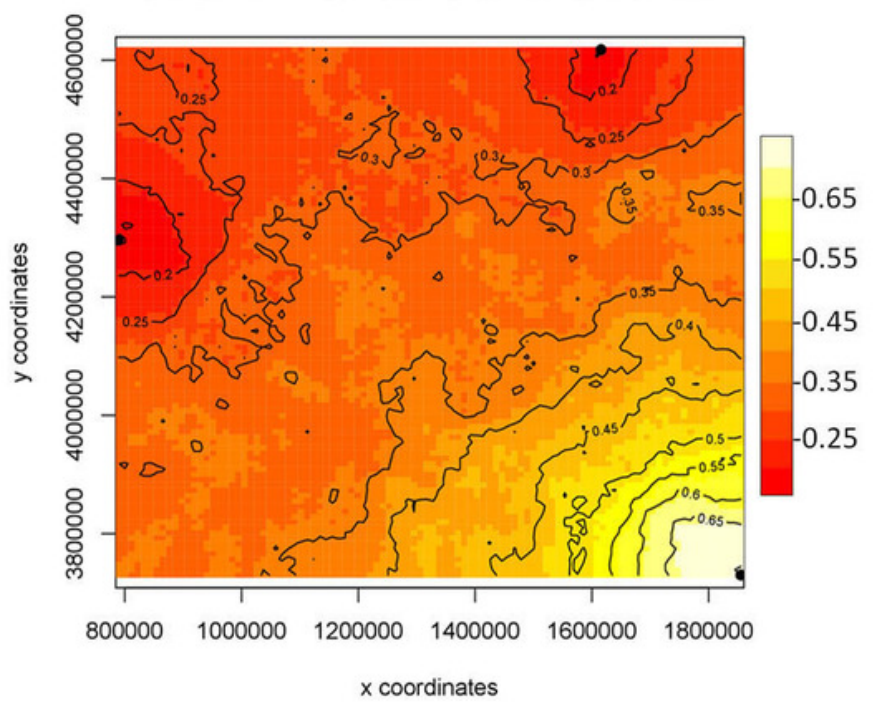

